\title{
Synthesis of Hydrophobic MIL-53(Al)-nanoparticles in Low Weight Alcohols: Systematic Investigation of Solvent Effects
}

\author{
Jan Warfsmann ${ }^{\mathrm{a}}$, Begum Tokay ${ }^{\mathrm{a}}$ and Neil R. Champness ${ }^{\mathrm{b}}$ * \\ a-Chemical and Environmental Engineering Department, Faculty of Engineering, University of Nottingham, \\ University Park, Nottingham NG7 2RD, United Kingdom \\ b-School of Chemistry, University Park, University of Nottingham, Nottingham, NG7 2RD, United Kingdom \\ Emails: Jan.Warfsmann@nottingham.ac.uk; Neil.Champness@nottingham.ac.uk;
}

Begum.Tokay@nottingham.ac.uk

\begin{abstract}
The effects of using low-weight alcohols, methanol and ethanol, for the synthesis of MIL-53(Al) are investigated and the results directly compared with analogous synthesis in water and $N, N$ dimethylformamide (DMF). When methanol is employed in the synthesis of MIL-53(Al), termed MIL-53(MeOH), several unique properties are observed. The breathing phenomenon which is known for MIL53(Al) derivatives, prepared using water or DMF as reaction solvent, is not observed for samples prepared from methanol and the framework adopts, and remains in, the large-pore form. Thus, measurement of $\mathrm{N}_{2}-$ isotherms and calculation of internal surface areas have verified that the synthesis of MIL-53(MeOH) leads to a product which is highly porous without the requirement for an energy-consuming activation process. Furthermore, X-ray diffraction measurements and scanning electron microscopy at different humidity levels reveal a reversible loss of crystallinity at high humidity levels for MIL-53(MeOH) which was not observed previously for any other known MIL-53 derivative. In contrast the synthesis of MIL-53(AI) in ethanol leads of a product with low crystallinity.
\end{abstract}

\section{Introduction:}

Metal-organic frameworks (MOFs) have received extensive interest due to their highly porous structure and their ability to act as efficient hosts for guest molecules. The possibility to tune pore dimensions and functionality has led to the application of $\mathrm{MOFs}^{1}$ in fields as diverse as gas adsorption ${ }^{2}$, drug delivery ${ }^{3}$, protein encapsulation $^{4}$, as crystal sponges ${ }^{5}$ or magnetic and electronic devices. ${ }^{6,7}$ The MIL-53 class (MIL = Matériaux de l'Institut Lavoisier) of materials received much attention in recent years due to their unusual structural behaviour. Ferey et.al reported the first chromium-containing MIL-53 ${ }^{8}$, formed by $\mathrm{CrO}_{4}\left(\mathrm{OH}_{2}\right)$ octahedral clusters connected by 1,4-benzenedicarboxylic acid ( $\left.\mathrm{H}_{2} \mathrm{BDC}\right)$ that lead to a porous material with one-dimensional rhombus-shaped channels. Numerous MIL-53 derivatives has been synthesized whilst preserving the overall structure by using different metal sources, e.g. Fe ${ }^{9,10}, \mathrm{Al}^{11}$, Ga ${ }^{12}$ and $\mathrm{Sc}^{13}$, and/or $\mathrm{BDC}^{2-}$ derivatives, e.g. amino ${ }^{14}$, fluorine ${ }^{15}$, chlorine, nitro, hydroxyl ${ }^{16}$ or carbamate ${ }^{17}$. The possibility to employ post-synthetic modification ${ }^{18}$ and using mixtures of metals ${ }^{19}$ or ligands ${ }^{20}$ in the same framework material further increase the variability of MIL-53. As a result, MIL-53 has been successfully applied in gas separation ${ }^{21}$ and storage ${ }^{22}$, heavy metal capture ${ }^{23}$, catalysis ${ }^{24,25}$ and controlled drug delivery ${ }^{26}$. Besides the synthetic variability, MIL-53 is particularly notable for the so called breathing effect ${ }^{11,27}$. MIL-53 has a flexible framework and the internal pore size and volume change by up to $33 \%{ }^{12,28}$ upon appropriate stimulation including guest molecule adsorption ${ }^{11}$ or temperature variation ${ }^{19}$. For the aluminium and chromium derivatives, small and large pore form ${ }^{11,18}$ (pore apertures are $0.26 \mathrm{~nm} \times 1.36 \mathrm{~nm}$ and $0.85 \mathrm{~nm} \times$ $0.85 \mathrm{~nm}$ respectively) are observed, while for the $\mathrm{Fe}^{29}$ and $\mathrm{Ga}^{12}$ derivatives several additional pore forms exists. The breathing of MIL-53 is most commonly caused by adsorption of water or $\mathrm{CO}_{2}$ and MIL-53 adsorbs 
water from ambient air within minutes even at low relative humidity of $10 \%{ }^{11,30,31}$. Although, the adsorption of water is desired for some applications e.g. water capture from ambient conditions for usage as drinking water ${ }^{32}$ or in heat pumps ${ }^{33}$, this feature can limit the use of MIL-53 for many industrial applications where adsorbed water has to be removed prior to further application. Due to the ubiquity of humidity in ambient air, several studies investigated the usage of additional agents or synthesis methods in order to increase the hydrophobicity of MIL-53, such as using ionic liquids ${ }^{31}$ or hydrophobic linkers ${ }^{34}$ during synthesis. However, possible trade-offs are increased synthesis times (up to one week) or a reduced pore volume. Although MIL-53 has been synthesized by microwave radiation ${ }^{35}$, ultrasound ${ }^{36}$ or in continuous flow conditions ${ }^{37}$, solvothermal synthesis at autogenous pressure and elevated temperature still remains the most common way to prepare MIL-53 employing $\mathrm{N}, \mathrm{N}$-Dimethylformamide (DMF) and water as solvents ${ }^{38}$. Synthesis of MIL-53 in DMF has the advantages of high product yields (up to 100\%) and the possible synthesis of nano-sized particles ( $<100 \mathrm{~nm}$ crystals), but DMF is flammable, toxic and can cause congenital disorders ${ }^{39}$. Moreover, the low DMF vapour pressure prevents the easy removal of excess/residue DMF (at $20{ }^{\circ} \mathrm{C}$ for DMF $0.377 \mathrm{kPa}$ in comparison with $2.33 \mathrm{kPa}$ for water and $12.9 \mathrm{kPa}$ for $\mathrm{MeOH}^{40}$ ) from the pores and for several MOFs, including MIL-53, coordination of DMF to the MOF metal nodes has been observed, which further complicates the purification/activation process ${ }^{41}$. Therefore, DMF usage in industrial scale synthesis is not recommended and alternatives should be sought ${ }^{42}$. Water would be a suitable alternative solvent, but low yields remain a significant challenge ${ }^{43}$. Another challenge is the difficulty to prepare nanosized particles in water, which is desirable for the use as catalyst, electrode material or medical application to name just a few ${ }^{44-46}$. Ahnfeld et.al ${ }^{47}$ have shown by a high throughout synthesis in low weight alcohols e.g. $\mathrm{MeOH}$ and $\mathrm{EtOH}$ that the synthesis MIL-53 of is in principle possible. These solvents have advantages such as low cost and ease of removal, making the synthesis of MIL-53 more sustainable. Although some studies investigate the synthesis of MIL-53 in water and DMF ${ }^{43,48}$, a wide range of applied synthesis conditions e.g. temperature, reaction time, concentration, metal-to-linker ratio and additives make the direct comparison of these results complicated ${ }^{11,48-52}$. To the best of our knowledge, no reports of the successful synthesis and characterisation of MIL-53 in simple alcohols have been reported previously. We present herein the MIL-53 synthesis in $\mathrm{MeOH}$ and $\mathrm{EtOH}$ to determine the influence of these solvents in comparison with MIL-53 synthesized in water and DMF. The MIL-53 aluminium derivative was used as the test compound due to the known lower toxicity in comparison with other derivative such as chromium ${ }^{16}$ and the commercial availability (as BasoliteA100 ${ }^{\circledR}$ ).

\section{Experimental}

Aluminium nitrate nonahydrate, $\mathrm{Al}\left(\mathrm{NO}_{3}\right)_{3} \cdot 9 \mathrm{H}_{2} \mathrm{O}$, and 1,4-benzenedicarboxylic acid (>98\%) were purchased from Sigma Aldrich. $\mathrm{N}, \mathrm{N}$-Dimethylformamide, $\mathrm{MeOH}, \mathrm{EtOH}$ and acetone were provided from FisherScientific. All chemicals were used without further purifications. Deionised water (DI-water) was collected from a Millipore Direct-Q 5 UV water purification system.

\section{Synthesis:}

MIL-53 was synthesized according to a modified synthesis protocol ${ }^{49}$. Briefly, a reaction mixture of $\mathrm{Al}\left(\mathrm{NO}_{3}\right)_{3} \cdot 9 \mathrm{H}_{2} \mathrm{O}(5.99 \mathrm{mmol} ; 2.246 \mathrm{~g})$ and $\mathrm{H}_{2} \mathrm{BDC}(5.38 \mathrm{mmol} ; 0.895 \mathrm{~g})$, was prepared in $30 \mathrm{ml}$ of solvent (DMF, water, $\mathrm{MeOH}$ or EtOH) and stirred vigorously at room temperature for $15 \mathrm{~min}$ and transferred to a $45 \mathrm{ml}$ Teflon lined stainless steel autoclave. The reaction mixture was heated to $150{ }^{\circ} \mathrm{C}$ for $5 \mathrm{~h}$ after which the product was separated by centrifugation ( $4500 \mathrm{rpm}$ for $30 \mathrm{~min}$ ). The solution was decanted and the crystalline material washed with DMF $(2 \times 50 \mathrm{ml})$ and acetone $(1 \times 50 \mathrm{ml})$. The product, a white powder, was dried at $150{ }^{\circ} \mathrm{C}$ for $17 \mathrm{~h}$. Unreacted starting material and residue solvent were removed from the as-synthesized samples (referred as MIL53-AsSyn subsequently) by heating at $333^{\circ} \mathrm{C}$ for 3 days (referred as MIL53-Acti subsequently) ${ }^{11}$. For simplification, the products are referred to as MIL-53( $\left.\mathrm{H}_{2} \mathrm{O}\right)$, MIL-53(DMF), MIL-53(MeOH) and MIL-53(EtOH) respectively. 


\section{Characterisation:}

X-Ray diffraction (XRD) measurement was performed using a PANanalytical X'Pert Pro diffractometer operated at $40 \mathrm{kV}$ and $40 \mathrm{~mA}$ and $\mathrm{Cu} \mathrm{K} \alpha$ radiation ( $\lambda=1.540598 \AA$ ) equipped with a PIXCell3D detector. The experiments were conducted in continuous scanning mode with the goniometer fixed in the theta/theta orientation. For the indecent site and detector site a soller slit of $0.04 \mathrm{rad}^{\circ}$ and an incident beam mask of $15 \mathrm{~mm}$ was used. The imitated length was $10.0 \mathrm{~mm}$. In a typical experiment $\sim 50 \mathrm{mg}$ of MIL-53 powder was used and scans were conducted in a range of $5^{\circ}-35^{\circ} 2 \theta$ with a step size of $0.006565^{\circ}$ and a scan time of $51 \mathrm{~s}$ per step., resulting in a total measurement time of $17 \mathrm{~min}$. For temperature dependant XRD measurements an AntonPaar HTK $1200 \mathrm{~N}$ oven was used. For all measurements, the sample was heated between $25^{\circ} \mathrm{C}$ and $250{ }^{\circ} \mathrm{C}$ (between $25^{\circ} \mathrm{C}$ and $200^{\circ} \mathrm{C}$ in $25^{\circ} \mathrm{C}$ steps with one additional measurement at $250{ }^{\circ} \mathrm{C}$ ) and then cooled down to $25^{\circ} \mathrm{C}$. Additional diffractograms at $25^{\circ} \mathrm{C}$ were measured immediately after reaching $25^{\circ} \mathrm{C}$ again ( 0 min waiting time) and after waiting times of $30 \mathrm{~min}$ and $60 \mathrm{~min}$ respectively. All samples for the temperature depended XRD measurement were kept in a saturated water atmosphere for 3 days prior to XRD measurements. Scanning Electron Microscopy (SEM) was used to characterise the morphology and crystal size (from an average of 25 particles). Measurements were conducted with JEOL JSM-7100F Field Emission Gun (FEG) instrument with a beam voltage of $15 \mathrm{kV}$. Samples were sputtered with a $10 \mathrm{~nm}$ iridium film by a Quorm Q150T ES coater in order to increase conductivity of the samples. Environmental SEM (ESEM) in varying relative humanity were measured using a FEI Quanta $650 \mathrm{ESEM}$ at $15 \mathrm{kV}$. The samples were not coated for ESEM measurements. Thermal stability was investigated using a TA instruments Q500 thermal gravimetric analyser. Samples were heated up to $700{ }^{\circ} \mathrm{C}$ under air flow $(100 \mathrm{ml} / \mathrm{min})$ with a heating rate of $5{ }^{\circ} \mathrm{C} / \mathrm{min}$. Sample porosity and BET area were determined by $\mathrm{N}_{2}$-adsoprtion via Micrometrics Tristar II-adsorber. Degassing of the samples were conducted in vacuum at $80^{\circ} \mathrm{C}$ for $1 \mathrm{~h}$ and at $150{ }^{\circ} \mathrm{C}$ overnight. $\mathrm{N}_{2}$-isotherms were measured at $77 \mathrm{~K}$. Surface area was calculated by BET-Theory using 7 data points between 0.05 and $0.28 \mathrm{p} / \mathrm{p} 0$. Water isotherms were measured with a Micrometrics 3Flex adsorber at $25^{\circ} \mathrm{C}$ between 0.1 and $0.9 \mathrm{p} / \mathrm{p0}$. For a typical experiment $100 \mathrm{mg}$ of sample was degassed at $150{ }^{\circ} \mathrm{C}$ in vacuum overnight before measurement of water isotherms. The water vapour of different relative humidity was created by bubbling dry nitrogen though deionized water. For every data point maximum time allowed to reach equilibrium (pressure change not more than $5 \%$ during $600 \mathrm{~s}$ ) was set to $16 \mathrm{~h}$. FT-IR spectroscopy was measured on a Bruker Alpha equipped with a Platinum ATR module. Spectra were measured with $4 \mathrm{~cm}^{-1}$ resolution collecting 50 consecutive measurements.

\section{Results and Discussions:}

\section{Investigation of morphology by SEM}

SEM images of powder samples synthesised using water, DMF and $\mathrm{MeOH}$ before and after activation are shown in Figure 1. When water is used as the solvent for synthesis, generating MIL-53 $\left(\mathrm{H}_{2} \mathrm{O}\right)$, micrometre sized $(\sim 3 \mu \mathrm{m})$ star-shaped particles are observed (Figure 1a+b), indicating intergrowth of orthorhombic crystals, the typical shape of MIL-53 single crystals ${ }^{53,54}$. After activation at high temperature, cracks and broken intergrown particles were observed (Figure $1 \mathrm{~b}$ ), presumably caused by thermal stress during the activation process.

In contrast to particles prepared in water, SEM images of MIL-53(DMF) reveal spherical particles with the size of $41 \pm 17 \mathrm{~nm}$, which agglomerate to form larger particles of several micrometre (See SI Figure S2a). This can be partly explained with the drying process for SEM imaging, which caused strong particle agglomeration ${ }^{55}$. In contrast to MIL-53 $\left(\mathrm{H}_{2} \mathrm{O}\right)$ samples, activation caused no cracks in the particles' surface (Figure 1c), possibly as a result of the smaller particles size of MIL-53(DMF). Using MeOH as solvent, two particle morphologies were observed: (1) spherical particles similar size to MIL-53(DMF) and (2) longer rodshaped particles with comparable diameters to the spherical particles but with substantially longer length of $178 \pm 42 \mathrm{~nm}$ (Figure 1d). As with MIL-53(DMF), a tendency towards agglomeration to micrometre sized 
particles was observed (see Figure S3a). Interestingly, in DMF and $\mathrm{MeOH}$, nanometre sized particles are prepared.

Previous investigations to explain the different particles sizes observed for MIL-53 prepared from water or DMF have identified the varying deprotonation rate of the linker $\mathrm{H}_{2} \mathrm{BDC}$ as the main reason for the behaviour in different solvents ${ }^{43}$. In these reactions the reaction solvent acts as a base. In general, a faster nucleation rate is expected to lead to the formation of smaller particles ${ }^{56}$. A good indicator for the basicity, and in this regard for the deprotonation rate, is the $\mathrm{pKa}$ of the solvents used (water: $14^{57}$; DMF: $-0.30^{58}$, $\mathrm{MeOH}=15.7^{58}$ ). However, this measure suggests that $\mathrm{MeOH}$ is slightly more acidic than water and should, in principle, lead to the formation of larger particles. We therefore assume that the solubility of the linker in the reaction solvents also has a significant effect on the particles size during the formation of MIL-53. At reaction temperature of $150{ }^{\circ} \mathrm{C}$, the linker only dissolves partly in water $\left(0.0065 \mathrm{~g} \mathrm{H}_{2} \mathrm{BDC}\right.$ in $100 \mathrm{~g}$ water $\mathrm{r}^{59}$ ) and react with metal ions to from MIL-53 seeds. We assume due to the shape of the MIL-53( $\left.\mathrm{H}_{2} \mathrm{O}\right)$ (see Figure 1 ), that in water the growth from already existing seeds is preferred over the nucleation of new seeds, which leads to the formation of particles exceeding several micrometres. In DMF ( $14.49 \mathrm{~g} \mathrm{H}_{2} \mathrm{BDC}$ in $100 \mathrm{~g}$ $\mathrm{DMF}^{60}$ ) and methanol $\left(2.9 \mathrm{~g} \mathrm{H}_{2} \mathrm{BDC}\right.$ in $\left.100 \mathrm{~g} \mathrm{MeOH}^{61}\right)$ the solubility is several magnitudes higher in comparison with water. The $\mathrm{BDC}^{2-}$ is therefore fully dissolved, leading to a greater number of seeds and hence more nanometre sized MIL-53 particles. The solubility might affect the yield as well, whereas for DMF a nearly complete conversion was observed, while the yields are in methanol (52\%) and water (18\%) significantly lower.

\section{Investigation of crystallinity and breathing effect of MIL-53 by XRD}

XRD pattern of samples of MIL-53 synthesised in water, DMF or MeOH before and after activation are shown in Figure 2, including a comparison to the pattern calculated from single crystal data ${ }^{11}$. Prior to activation, MIL-53 $\left(\mathrm{H}_{2} \mathrm{O}\right)$ has a pattern which is in good agreement with the calculated XRD pattern of MIL-53-AsSyn. During the synthesis $\mathrm{H}_{2} \mathrm{BDC}$ remains in the sample pores ensuring the large pore form. Due to interaction between the $\mathrm{H}_{2} \mathrm{BDC}$ and the pore walls, the pore diameter is slightly smaller $(0.73 \mathrm{~nm} \times 0.77 \mathrm{~nm})$ in comparison with the activated large pore from ${ }^{11}(0.85 \mathrm{~nm} \times 0.85 \mathrm{~nm})$, leading to a shift of the peaks in the XRD pattern (e.g. the peak in the calculated pattern at $2 \theta=8.94^{\circ}$ shift to $8.72^{\circ}$ ). Activated MIL-53 $\left(\mathrm{H}_{2} \mathrm{O}\right)$ adsorbs water from ambient air, leading to shrinkage of the pores $(0.26 \mathrm{~nm} \times 1.36 \mathrm{~nm})$. Both forms can be easily distinguished by XRD, where the large pore form shows a characteristic peak at $\sim 15^{\circ}$ while the small pore form is indicated by a peak at $\sim 12^{\circ}$. The pattern of MIL53(DMF)-AsSyn matches the calculated As-Syn pattern. The observed broader peaks are in agreement with the observed smaller particle size of MIL-53(DMF) in comparison with MIL-53( $\left.\mathrm{H}_{2} \mathrm{O}\right)$. After activation, these peaks were shifted to smaller $2 \theta$ values, showing the removal of $\mathrm{H}_{2} \mathrm{BDC}$. The similar intensities of the characteristic peaks at $12^{\circ}$ and $15^{\circ}$ may suggest equal proportions of small and large pores in the sample, respectively. In contrast to MIL-53( $\left.\mathrm{H}_{2} \mathrm{O}\right)$ and MIL-53(DMF), no peak at $2 \theta=12^{\circ}$ is observed in the pattern of MIL-53(MeOH) indicating the absence of the small pore form. The characteristic peaks are already in good agreement with an activated sample. Furthermore, the peak positions of MIL-53(MeOH) have not shifted to lower $2 \theta$ values after activation. This imply an activated MIL-53 sample.

The measurement of the internal pore volume by BET help to further confirm these assumptions. The measured BET-area of the activated samples prepared in water and DMF show the formation of porous, crystalline samples and are with $1002 \mathrm{~m}^{2} / \mathrm{g}$ for the water sample and $1084 \mathrm{~m}^{2} / \mathrm{g}$ for the DMF sample in good agreement with previously reported data ${ }^{11,21,62}$. The measured BET surface area of MIL-53(MeOH) $\left(895 \mathrm{~m}^{2} / \mathrm{g}\right)$ is slightly lower than the samples prepared in water and DMF. However, in case of MIL-53(MeOH) only a small difference between the as-synthesized sample and the activated sample regarding surface area and microporosity ( $10 \%$ for both) was observed (see Table 1). For MIL-53(DMF), the differences regarding surface area $(\sim 29 \%)$ and microporosity $(\sim 49 \%)$ are larger and for MIL-53 $\left(\mathrm{H}_{2} \mathrm{O}\right)$-AsSyn no porosity could be measured. This may indicate, combined with the results of the XRD measurements, that nearly no $\mathrm{H}_{2} \mathrm{BDC}$ is trapped in $\mathrm{MIL}-53(\mathrm{MeOH})$ after the reaction and milder or even no 
activation might be necessary to access the framework porosity. A MIL-53 derivate synthesises using EtOH as solvent revealed similar particles size and morphology to MIL-53(MeOH) (see Figure S5). Characteristic peaks in XRD at $2 \theta=8.48^{\circ}, 15.20^{\circ}$ and $17.34^{\circ}$ indicate the synthesis of MIL-53 (see Figure S6). However, the greatly reduced intensity in comparison with the samples prepared in water, DMF and $\mathrm{MeOH}$ imply the synthesis of a sample with low crystallinity, verifying the observation of Ahnfeld et al. ${ }^{18}$. Furthermore, after activation, characteristic diffraction peaks were not observed anymore and an amorphous product was obtained. Therefore no further investigation of MIL-53(EtOH) was conducted in this research.

\section{Investigation of the breathing effect and water adsorption kinetics}

As already mentioned, the XRD pattern of MIL-53 helps to distinguish between the small and large pore form. The comparison between the As-Syn and activated samples of MIL-53 imply a different water adsorption kinetic and breathing phenomenon depending on the used solvent for the synthesis. To further investigate this observation, temperature depended PXRD was conducted. Samples were saturated in water vapour and XRD pattern measured at different temperature to investigate the removal of water from the pores and consequently the breathing phenomenon. Figure 3a show the temperature depended XRD pattern of MIL-53( $\left.\mathrm{H}_{2} \mathrm{O}\right)$-Acti. Only a peak at $2 \theta=12^{\circ}$ is visible at $25^{\circ} \mathrm{C}$ and the sample is found to be solely in the small pore form. At approximately $75^{\circ} \mathrm{C}$ the small pore peak becomes broader and decrease in intensity, while the large pore peak at $15^{\circ}$ increase in intensity. At $150^{\circ} \mathrm{C}$, only the large pore form was observed. After cooling down to $25^{\circ} \mathrm{C}$ again, the intensity of the small pore peak at $12^{\circ}$ increased whereas the intensity of the large pore $15^{\circ}$ peak decreased with every consecutive waiting step, showing fast water adsorption from ambient air.

As shown in Figure 3b, MIL-53(DMF) sample structure did not change completely into the small pore form after being exposed to saturated water atmosphere. Instead the XRD pattern indicate a 1:1 ratio of large pore to small pore based on the peak intensities. During the heating, the small pore peak at $12.51^{\circ}$ shifts to larger $2 \theta$ values when temperature was increased from $25^{\circ} \mathrm{C}$ to $100^{\circ} \mathrm{C}$ with a shift up to $0.64^{\circ}$. For higher temperature, this peak shifts back to the initial position while reducing in intensity until loss of the peak at $200^{\circ} \mathrm{C}$. After cooling down the sample back to $25^{\circ} \mathrm{C}$ a small peak at $12.49^{\circ}$ appeared after $1 \mathrm{~h}$ period. This and the 1:1 ratio between small and large pore form in the starting material, suggest slower water adsorption in comparison with MIL-53( $\left.\mathrm{H}_{2} \mathrm{O}\right)$ sample. In contrast, in the XRD pattern of MIL-53(MeOH)-Acti (Figure 3c) a small pore peak at $2 \theta=\sim 12^{\circ}$ could be not observed, which implies that MIL-53(MeOH) does not show any breathing phenomenon at all. For the initial measurement at $25^{\circ} \mathrm{C}$, the observed peaks were considerably lower in intensity than for MIL-53(Water)-Acti and MIL-53(DMF)-Acti. In order to understand this effect Environmental SEM (ESEM) measurements were employed. At higher humidity levels (>80\%), the particles start to fuse (see Figure 4), which might lead to a lower crystallinity of the sample and could be not observed for MIL-53(DMF) which has a similar morphology (see Figure 5). However, the temperature depended SEM show that, in contrast to moisture sensitive MOFs like HKUST-1 or MOF- $53^{30}$, the crystallinity of MIL-53(MeOH) is restored after the removal of water.

The measurement FTIR spectra of the activated sample further confirm this observation (see Figure S7). MIL-53 samples were dried at $125^{\circ} \mathrm{C}$ and IR spectra measured 5 min after removal from oven. IR spectra of all samples show peaks at $1414 \mathrm{~cm}^{-1}$ and $1510 \mathrm{~cm}^{-1}$, which are attributed to the symmetrical and asymmetrical stretching of the framework carboxylate groups ${ }^{63}$.Peaks at $1120 \mathrm{~cm}^{-1}, 3463 \mathrm{~cm}^{-1}$ and $3610 \mathrm{~cm}^{-1}$ are only observed in the IR spectrum of MIL-53( $\left.\mathrm{H}_{2} \mathrm{O}\right)$, which indicate the adsorption of water. Figure 6 shows water isotherms for the activated samples MIL-53( $\left.\mathrm{H}_{2} \mathrm{O}\right)$, MIL-53(DMF) and MIL-53(MeOH) and verify different hydrophobicity of the samples. The MIL-53 derivative prepared in water shows a fast uptake of water at low relative pressure of $0.1 \mathrm{p} / \mathrm{p} 0$ and reach a plateau at $5 \mathrm{mmol} / \mathrm{g}$ adsorbed water. The isotherm has Type-l isotherm shape. This imply strong interaction between the adsorptive and adsorbent at low relative pressure and a sample without macroporosity. The pores contract into the small pore form, preventing further water adsorption. Both MIL-53(DMF) and MIL-53(MeOH) show at lower relative pressure only a low water uptake followed by a sharp increase at $0.5 \mathrm{p} / \mathrm{p} 0$ for $\mathrm{MIL}-53(\mathrm{MeOH})$ and at $0.6 \mathrm{p} / \mathrm{p} 0$ 
for MIL-53(DMF). At low relative pressure, the water uptake of MIL-53(MeOH) is lower than MIL53(DMF).this tendency reserve at higher relative pressure. At $0.9 \mathrm{p} / \mathrm{p0}$ for MIL-53(DMF), a total uptake of $10 \mathrm{mmol} / \mathrm{g}$ was measured while the total uptake of MIL-53(MeOH) is with $22 \mathrm{mmol} / \mathrm{g}$ much higher. The water isotherms resemble for both samples a Type-V isotherm, which indicates weak interaction at low relative pressure following with clustering of water molecules (pore condensation) at higher relative pressure. Above $0.8 \mathrm{p} / \mathrm{p} 0$ a further increase of water uptake indicates water adsorption in the space between the particles.

Similar trends for water adsorption for MIL-53 samples were already observed. Mounfield and Walton ${ }^{48}$ confirmed a dependency between reaction temperature and breathing phenomenon for MIL-53(DMF). MIL-53(DMF) prepared at $120^{\circ} \mathrm{C}$ does not show any breathing phenomenon while the $220^{\circ} \mathrm{C}$ sample show a slight breathing of the pores. Furthermore at low relative water pressure $(<0.5 p / p 0)$ the samples show lower water uptake $\left.(1.5 \mathrm{mmol} / \mathrm{g} \text { for DMF samples in comparison with } 4 \mathrm{mmol} / \mathrm{g} \text { for MIL-56(H2O) })^{48}\right)$. The increased hydrophobicity was attributed to remaining DMF molecules in the framework. In this study, activation was conducted at elevated temperature to ensure the complete removal of any residue and TGA measurement (see Figure S8) have verified the absence of DMF in the samples. The absence of weight loss steps between $100{ }^{\circ} \mathrm{C}$ and $250^{\circ} \mathrm{C}$ (residue of $\mathrm{DMF}^{48}$ ) or $275^{\circ} \mathrm{C}$ and $420^{\circ} \mathrm{C}$ (residue of $\mathrm{H}_{2} \mathrm{BDC}^{11}$ ) indicate the formation of a product free of any residual species in the pores. Interestingly, the thermal stability of MIL-53(DMF) and MIL-53(MeOH) $\left(\sim 460^{\circ} \mathrm{C}\right)$ was slightly reduced in comparison with MIL-53(water) $\left(\sim 500^{\circ} \mathrm{C}\right)$. This may be due to the smaller particle size of MIL-53(MeOH) and MIL-53(DMF) in comparison with MIL-53( $\left.\mathrm{H}_{2} \mathrm{O}\right)$. Even so, the thermal stability of MIL-53 prepared in DMF or MeOH is higher in comparison with other commercial available, carboxylate containing MOFs like HKUST-1 $\left(240^{\circ} \mathrm{C}^{65}\right)$, or MOF-177 $\left(400^{\circ} \mathrm{C}^{66}\right)$. The varying hydrophobicity and breathing effect seems to be therefore an intrinsic characteristic of the MIL-53 derivates and can be controlled by the usage of different solvents. As a side note, MIL-53(DMF) seems to have an even higher hydrophobicity than MIL-53(MeOH) (increase of water uptake at $0.6 \mathrm{p} / \mathrm{p} 0$ in comparison with $0.5 \mathrm{p} / \mathrm{p0}$ in the water isotherms), but decomposes in high humidity. After measurement of water isotherm, the porosity of MIL-53(DMF) (see Figure S12) (BET area: $1084 \mathrm{~m}^{2} / \mathrm{g} \rightarrow$ $\left.377 \mathrm{~m}^{2} / \mathrm{g}\right)$ is greatly reduced, while only a small difference of $\sim 4 \%$ for MIL-53(MeOH) $\left(895 \mathrm{~m}^{2} / \mathrm{g} \rightarrow 862 \mathrm{~m}^{2} / \mathrm{g}\right)$ was observed. The porosity of MIL-53(water) has even slightly increased from $1002 \mathrm{~m}^{2} / \mathrm{g}$ to $1021 \mathrm{~m}^{2} / \mathrm{g}$. (see Table S15).

\section{Conclusion:}

We have successfully prepared MIL-53 in the low weight alcohol MeOH. This leads to the formation of a sample with nano-sized particles and compared the properties with MIL-53 prepared in water and DMF. The sample prepared in $\mathrm{MeOH}$ show a high porosity and thermal stability, which is typical for the MIL-53 class. In DMF and $\mathrm{MeOH}$, the solubility of $\mathrm{H}_{2} \mathrm{BDC}$ at the reaction temperature is several magnitudes higher in comparison with water, which leads to faster nucleation and crystal growth. This results in the formation of nanometer-sized particles. Further, MIL-53(MeOH) does not show no breathing effects, which can be partly attributed to the increased hydrophobicity of the sample. Interestingly, MIL-53(MeOH) particles have a tendency to fuse at high humidity. However in contrast to moisture sensitive MOFs like MOF-5, the framework of MIL-53(MeOH) does not collapse and the crystallinity of MIL-53(MeOH) will be restored after removal of water. Furthermore has the measurement of the internal surface area confirmed the synthesis of highly porous MIL-53(MeOH) even without a time and energy consuming activation process. Further research has to be done to understand, why the formation of MIL-53 was possible in $\mathrm{MeOH}$ but not in EtOH even though both solvents have similar chemical properties. 


\section{Acknowledgement:}

This work was supported by the Engineering and Physical Sciences Research Council (EPSRC) [under grant EP/L022494/1] and the University of Nottingham and the authors thank the Nanoscale and Microscale Centre (nmRC) for providing access to instrumentation and Ms. Nicola J. Weston for assistance with ESEM. Furthermore, the authors thank Prof. Dr. Michael Wark and Sven Warfsmann (Technical Chemistry, Institute for Chemistry, Carl-von-Ossietzky Universität Oldenburg) for measurement of $\mathrm{N}_{2}$ - and water-isotherms.

\section{References}

1 R. Ricco, C. Pfeiffer, K. Sumida, C. J. Sumby, P. Falcaro, S. Furukawa, N. R. Champness and C. J. Doonan, CrystEngComm, 2016, 18, 6532-6542.

2 J.-R. Li, R. J. Kuppler and H.-C. Zhou, Chem. Soc. Rev., 2009, 38, 1477-1504.

3 R. C. Huxford, J. Della Rocca and W. Lin, Curr. Opin. Chem. Biol., 2010, 14, 262-268.

4 X. Lian, Y. Fang, E. Joseph, Q. Wang, J. Li, S. Banerjee, C. Lollar, X. Wang and H.-C. Zhou, Chem. Soc. Rev., 2017, 46, 3386-3401.

5 W. M. Bloch, N. R. Champness and C. J. Doonan, Angew. Chem., 2015, 54, 12860-12867.

6 V. Stavila, A. A. Talin and M. D. Allendorf, Chem. Soc. Rev., 2014, 43, 5994-6010.

7 R. Ricco, L. Malfatti, M. Takahashi, A. J. Hill and P. Falcaro, J. Mater. Chem. A, 2013, 1, 13033.

8 G. Férey, M. Latroche, C. Serre, F. Millange, T. Loiseau and A. Percheron-Guégan, Chem. Commun., 2003, 2976-2977.

9 T. Devic, F. Salles, S. Bourrelly, B. Moulin, G. Maurin, P. Horcajada, C. Serre, A. Vimont, J.-C. Lavalley, H. Leclerc, G. Clet, M. Daturi, P. L. Llewellyn, Y. Filinchuk and G. Férey, J. Mater. Chem., 2012, 22, 10266.

10 F. Millange, N. Guillou, R. I. Walton, J.-M. Greneche, I. Margiolaki and G. Ferey, Chem. Commun., 2008, 4732-4734.

11 T. Loiseau, C. Serre, C. Huguenard, G. Fink, F. Taulelle, M. Henry, T. Bataille and G. Ferey, Chem. Eur. J., 2004, 10, 1373-1382.

12 C. Volkringer, T. Loiseau, N. Guillou, G. Ferey, E. Elkaim and A. Vimont, Dalton Trans., 2009, 22412249.

13 J. P.S. Mowat, S. R. Miller, A. M.Z. Slawin, V. R. Seymour, S. E. Ashbrook and P. A. Wright, Micropor. Mesopor. Mater., 2011, 142, 322-333.

14 J. Gascon, U. Aktay, M. Hernandez-Alonso, G. van Klink and F. Kapteijn, J. Catal, 2009, 261, 75-87.

15 L. Liu, X. Wang and A. J. Jacobson, Dalton Trans., 2010, 39, 1722-1725.

16 S. Biswas, T. Ahnfeldt and N. Stock, Inorg. Chem., 2011, 50, 9518-9526.

17 J. Wack, R. Siegel, T. Ahnfeldt, N. Stock, L. Mafra and J. Senker, J. Phys. Chem. C, 2013, 117, 1999120001.

18 T. Ahnfeldt, D. Gunzelmann, T. Loiseau, D. Hirsemann, J. Senker, G. Ferey and N. Stock, Inorg. Chem., 2009, 48, 3057-3064.

19 M. Mendt, B. Jee, N. Stock, T. Ahnfeldt, M. Hartmann, D. Himsl and A. Pöppl, J. Phys. Chem. C, 2010, 114, 19443-19451.

20 J. Yang, X. Yan, T. Xue and Y. Liu, RSC Adv, 2016, 6, 55266-55271.

21 S. Pourebrahimi, M. Kazemeini, E. Ganji Babakhani and A. Taheri, Microrpor. Mesopor. Mater., 2015, 218, 144-152.

22 K. Akhbari and A. Morsali, Mater. Lett., 2015, 141, 315-318.

23 T. A. Vu, G. H. Le, C. D. Dao, L. Q. Dang, K. T. Nguyen, Q. K. Nguyen, P. T. Dang, H. T. K. Tran, Q. T. Duong, T. V. Nguyen and G. D. Lee, RSC Adv, 2015, 5, 5261-5268.

24 J. Yan, S. Jiang, S. Ji, D. Shi and H. Cheng, Sci. China Chem., 2015, 58, 1544-1552.

25 U. Ravon, G. Chaplais, C. Chizallet, B. Seyyedi, F. Bonino, S. Bordiga, N. Bats and D. Farrusseng, Chem. Cat. Chem., 2010, 2, 1235-1238. 
26 P. Horcajada, T. Chalati, C. Serre, B. Gillet, C. Sebrie, T. Baati, J. F. Eubank, D. Heurtaux, P. Clayette, C. Kreuz, J.-S. Chang, Y. K. Hwang, V. Marsaud, P.-N. Bories, L. Cynober, S. Gil, G. Ferey, P. Couvreur and R. Gref, Nat. Mater., 2010, 9, 172-178.

27 M. Alhamami, H. Doan and C.-H. Cheng, Materials, 2014, 7, 3198-3250.

28 A. Schneemann, V. Bon, I. Schwedler, I. Senkovska, S. Kaskel and R. A. Fischer, Chem. Soc. Rev., 2014, 43, 6062-6096.

29 F. Millange, N. Guillou, M. E. Medina, G. Férey, A. Carlin-Sinclair, K. M. Golden and R. I. Walton, Chem. Mater., 2010, 22, 4237-4245.

30 J. Canivet, A. Fateeva, Y. Guo, B. Coasne and D. Farrusseng, Chem. Soc. Rev., 2014, 43, 5594-5617.

31 J. Liu, F. Zhang, X. Zou, G. Yu, N. Zhao, S. Fan and G. Zhu, Chem. Comm., 2013, 49, 7430-7432.

32 H. Kim, S. Yang, S. R. Rao, S. Narayanan, E. A. Kapustin, H. Furukawa, A. S. Umans, O. M. Yaghi and E. N. Wang, Science, 2017, 256, 430-434.

33 C. Janiak and S. K. Henninger, Chimia, 2013, 67, 419-424.

34 S. Biswas, T. Remy, S. Couck, D. Denysenko, G. Rampelberg, J. F. M. Denayer, D. Volkmer, C. Detavernier and P. van der Voort, Phys. Chem. Chem. Phys., 2013, 15, 3552-3561.

35 A. Laybourn, J. Katrib, R. S. Ferrari-John, C. G. Morris, S. Yang, O. Udoudo, T. L. Easun, C. Dodds, N. R. Champness, S. W. Kingman and M. Schröder, J. Mater. Chem. A, 2017, 5, 7333-7338.

36 E. Haque, N. A. Khan, J. H. Park and S. H. Jhung, Chemistry, 2010, 16, 1046-1052.

37 P. A. Bayliss, I. A. Ibarra, E. Pérez, S. Yang, C. C. Tang, M. Poliakoff and M. Schröder, Green Chem., 2014, 16, 3796.

38 N. Stock and S. Biswas, Chem. Rev., 2012, 112, 933-969.

39 C. A. Redlich, W. S. Beckett, J. Sparer, K. W. Barwick, C. A. Riely, H. Miller, Sigal, Steohen L., Shalat, Stuard L. and M. R. Cullen, Ann Intern Med, 1988, 108, 680-686.

40 W. M. Haynes, CRC handbook of Chemistry and Physics, (2016) 96th Edition, Boca Raton, 2016.

41 E. Tynan, P. Jensen, P. E. Kruger and A. C. Lees, Chem. Comm., 2004, 776-777.

42 D. Prat, O. Pardigon, H.-W. Flemming, S. Letestu, V. Ducandas, P. Isnard, E. Guntrum, T. Senac, S. Ruisseau, P. Cruciani and P. Hosek, Org. Process Res. Dev., 2013, 17, 1517-1525.

43 X. Cheng, A. Zhang, K. Hou, M. Liu, Y. Wang, C. Song, G. Zhang and X. Guo, Dalton Trans., 2013, 42, 13698-13705.

44 W. Lin, W. J. Rieter and K. M. L. Taylor, Angew. Chem., 2009, 48, 650-658.

45 M. Sindoro, N. Yanai, A.-Y. Jee and S. Granick, Acc. Chem. Res., 2014, 47, 459-469.

46 A. Majedi, F. Davar and A. R. Abbasi, Int. J. Nano Dimens., 2016, 7, 1-14.

47 T. Ahnfeldt, N. Guillou, D. Gunzelmann, I. Margiolaki, T. Loiseau, G. Ferey, J. Senker and N. Stock, Angew. Chem. Int.Ed., 2009, 48, 5163-5166.

48 W. P. Mounfield III and K. S. Walton, J. Collod Interface Sci., 2015, 447, 33-39.

49 H. Fan, H. Xia, C. Kong and L. Chen, Int. J. Hydrogen Ener., 2013, 38, 10795-10801.

50 C. Scherb, A. Schödel and T. Bein, Angew. Chem Int. Ed.., 2008, 120, 5861-5863.

51 L. Alaerts, M. Maes, L. Giebeler, P. A. Jacobs, J. A. Martens, J. F. M. Denayer, C. E. A. Kirschhock and D. E. de Vos, J. Am. Chem. Soc., 2008, 130, 14170-14178.

52 T. K. Trung, P. Trens, N. Tanchoux, S. Bourrelly, P. L. Llewellyn, S. Loera-Serna, C. Serre, T. Loiseau, F. Fajula and G. Ferey, J. Am. Chem. Soc., 2008, 130, 16926-16932.

53 F. Zhang, X. Zou, F. Sun, H. Ren, Y. Jiang and G. Zhu, CrystEngComm, 2012, 14, 5487-5492.

54 X. Qian, Z. Zhong, B. Yadian, J. Wu, K. Zhou, J. S.-k. Teo, L. Chen, Y. Long and Y. Huang, Int. J. Hydro. Energy, 2014, 39, 14496-14502.

55 S. Sorribas, B. Zornoza, P. Serra-Crespo, J. Gascon, F. Kapteijn, C. Téllez and J. Coronas, Micropor. Mesopor. Mater., 2016, 225, 116-121.

56 A. S. Myerson, ed., Handbook of Industrial Crystallization, Butterworth-Heinemann, Boston, 2nd edn., 2002.

57 Dougherty, Ralph, C. and L. N. Howard, J. Chem. Phys, 1988, 109, 7379-7393.

58 D. D. Perrin, Dissociation Constants of Organic Acids and Bases, Butterworths, London, 1965. 
59 A. Apelblat, E. Manzurola and N. Abo Balal, J. Chem. Thermodyn, 2006, 38, 565-571.

60 P. Ma and M. Chen, Chinese J. Eng., 2003, 11, 334-337.

61 A. Seidel, R. E. Kirk and D. F. Othmer, eds., Kirk-Othmer Encyclopedia of Chemical Technology, Wiley-Interscience, Hoboken, NJ, 4th edn., 1991-1996.

62 C.-X. Yang, S.-S. Liu, H.-F. Wang, S.-W. Wang and X.-P. Yan, Analyst, 2012, 137, 133-139.

63 J. M. Salazar, G. Weber, J. M. Simon, I. Bezverkhyy and J. P. Bellat, J. Chem. Phys., 2015, 142, 124702.

64 M. Thommes, K. Kaneko, A. V. Neimark, J. P. Olivier, F. Rodriguez-Reinoso, J. Rouquerol and K. S.W. Sing, Pure Appl. Chem., 2015, 87, 1-19.

65 S. S.-Y. Chui, S. M.-F. Lo, J. P. H. Charmant, A. G. Orpen and I. D. Williams, Science, 1999, 283, 11481150.

66 D. Saha and S. Deng, J. Phys. Chem. Lett., 2010, 1, 73-78. 

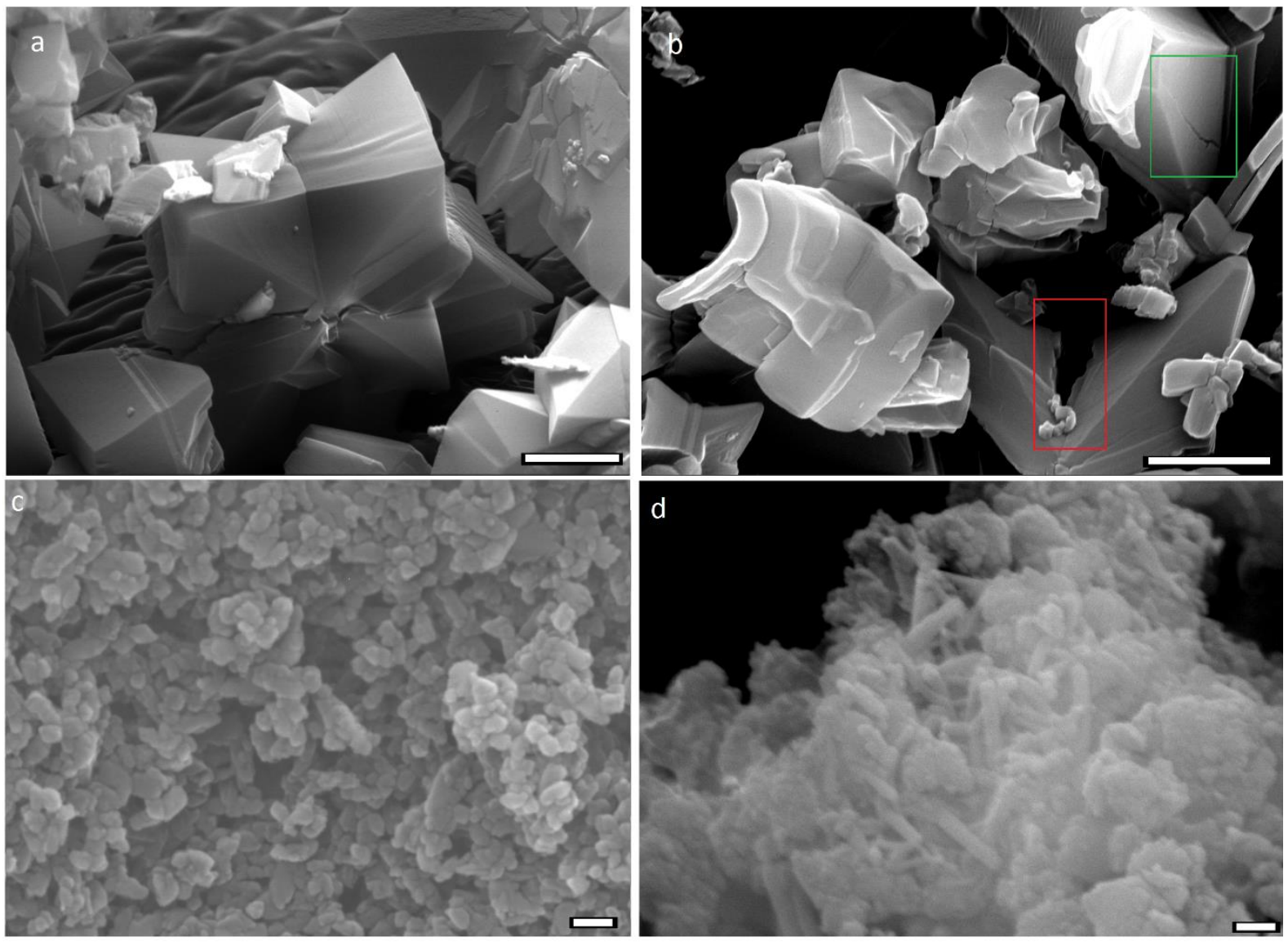

Figure 1: Comparison of SEM images from the sample MIL-53( $\left.\mathrm{H}_{2} \mathrm{O}\right)$-AsSyn (upper left, a), MII-53( $\left.\mathrm{H}_{2} \mathrm{O}\right)$-Acti (upper right, b), MIL53(DMF)-Acti (lower left, c) and MIL-53(MeOH)-Acti (lower right, d). For the MIL-53(water)-Acti sample intergrown star-shaped particles are identified with size of $3000 \mathrm{~nm}$. After activation by calcination cracks (identified by green rectangle) and breaking of the intergrown crystals could be observed (red rectangle). Scale bar in a and b:1 $\mu \mathrm{m}$; Scale bar in c and d: $100 \mathrm{~nm}$ 


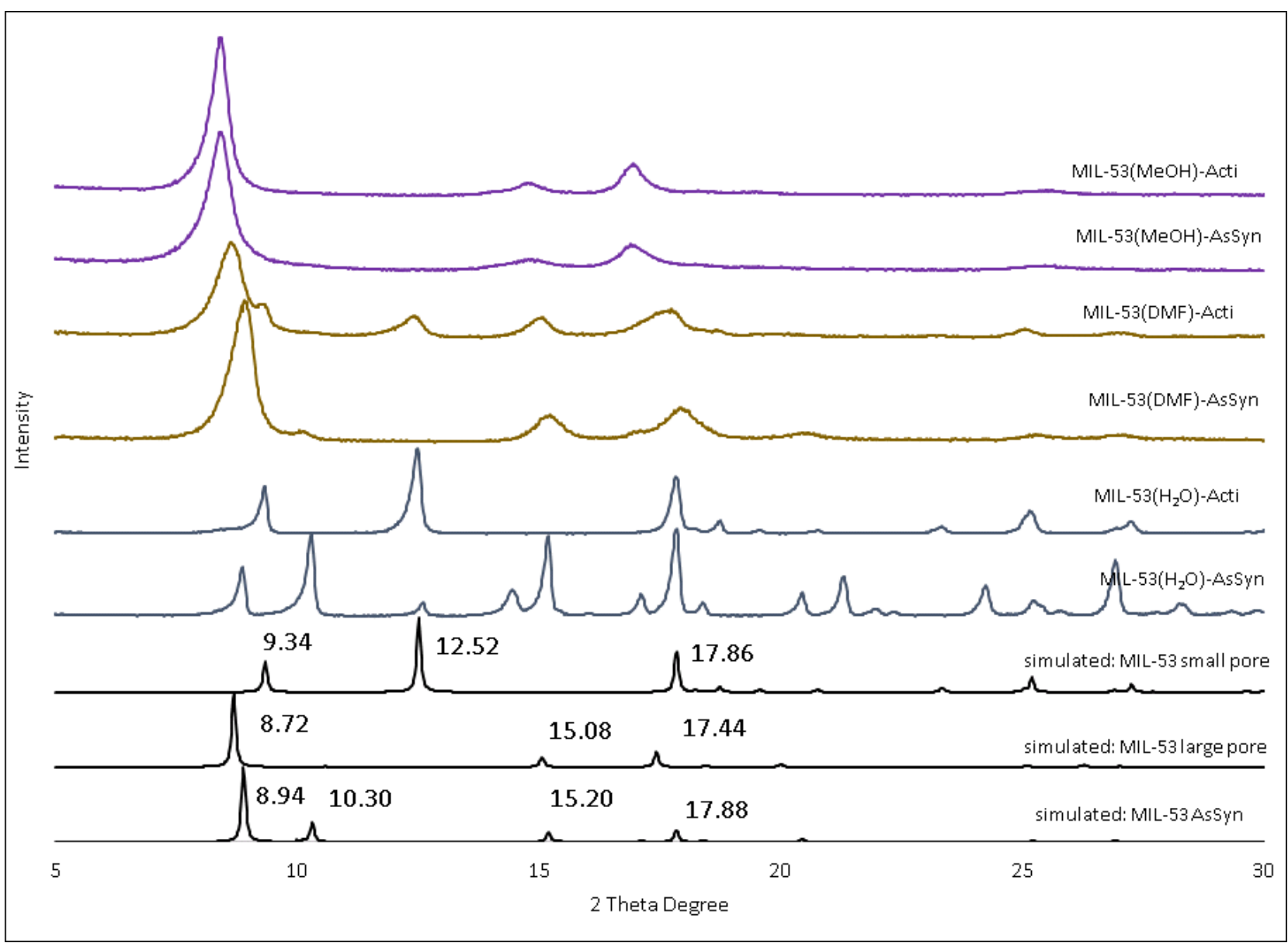

Figure 2: XRD pattern of as-synthesized and activated MIL-53 synthesized in water, DMF and MeOH. For comparison, XRD pattern of BDC containing MIL-53-AsSyn, large- and small pore from of MIL-53 are simulated from existing crystal data

Table 1: Compassion of BET-area and microporosity of the prepared MIL53 samples before and after activation. For MIL53( $\left.\mathrm{H}_{2} \mathrm{O}\right)$-AsSyn, no porosity could be measured.

\begin{tabular}{|c|c|c|}
\hline Compound & BET-area $\left(\mathrm{m}^{2} / \mathrm{g}\right)$ & t-plot micropore Volume $\left(\mathrm{cm}^{3} / \mathrm{g}\right)$ \\
\hline MIL-53( $\left.\mathrm{H}_{2} \mathrm{O}\right)-\mathrm{AsSyn}$ & / & / \\
\hline MIL-53( $\left.\mathrm{H}_{2} \mathrm{O}\right)-\mathrm{Acti}$ & 1002 & 0.44 \\
\hline MIL-53(DMF)-AsSyn & 767 & 0.23 \\
\hline MIL-53(DMF)-Acti & 1084 & 0.45 \\
\hline MIL-53(MeOH)-AsSyn & 805 & 0.35 \\
\hline MIL-53(MeOH)-Acti & 895 & 0.39 \\
\hline
\end{tabular}



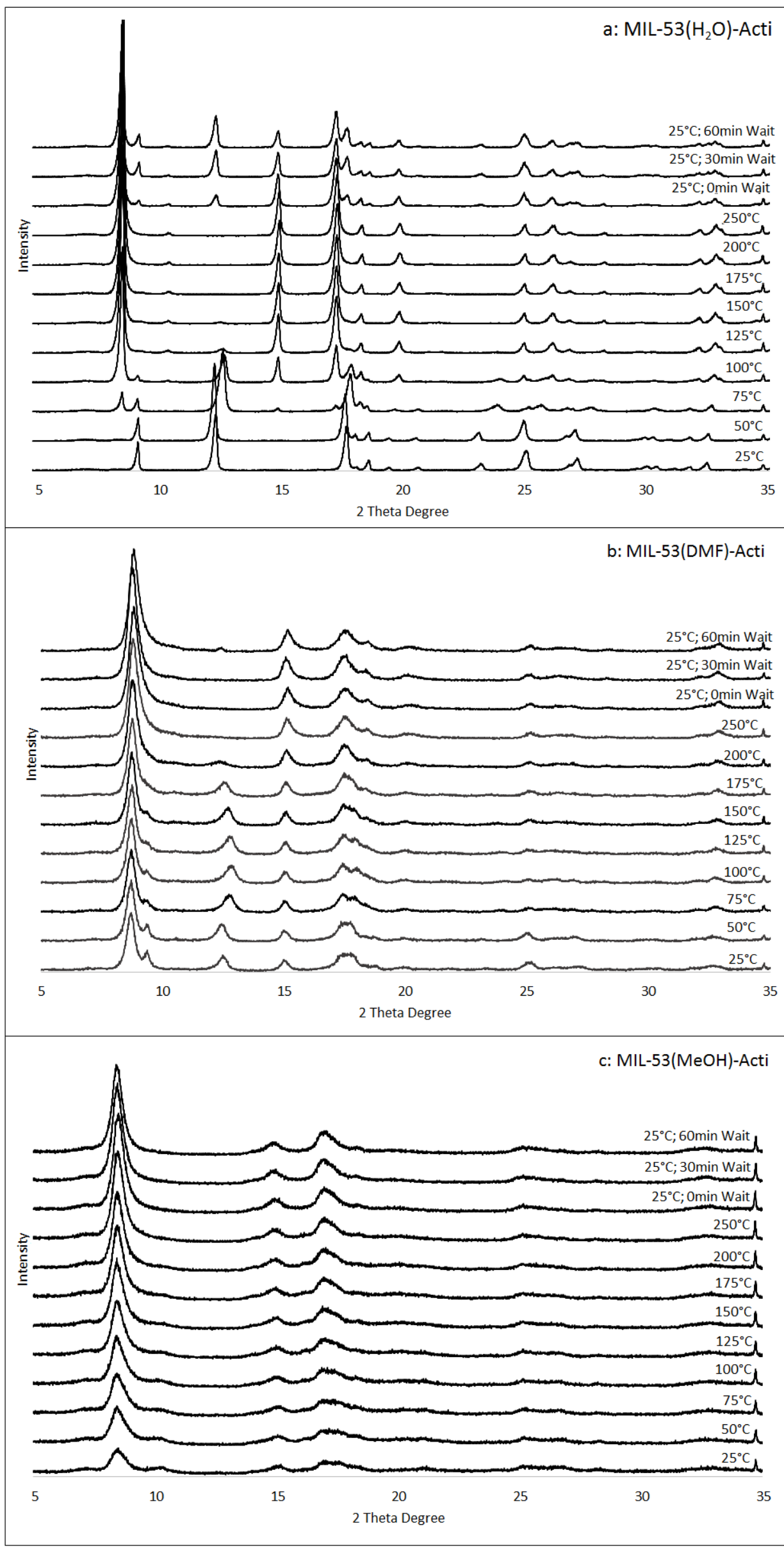

Figure 3: XRD patterns of a) MIL-53( $\left.\mathrm{H}_{2} \mathrm{O}\right)$-Acti, b) MIL-53(DMF)-Acti and c) MIL-53 (MeOH)-Acti measured at varying temperatures. All samples were saturated with water before measurement of the XRD series. 


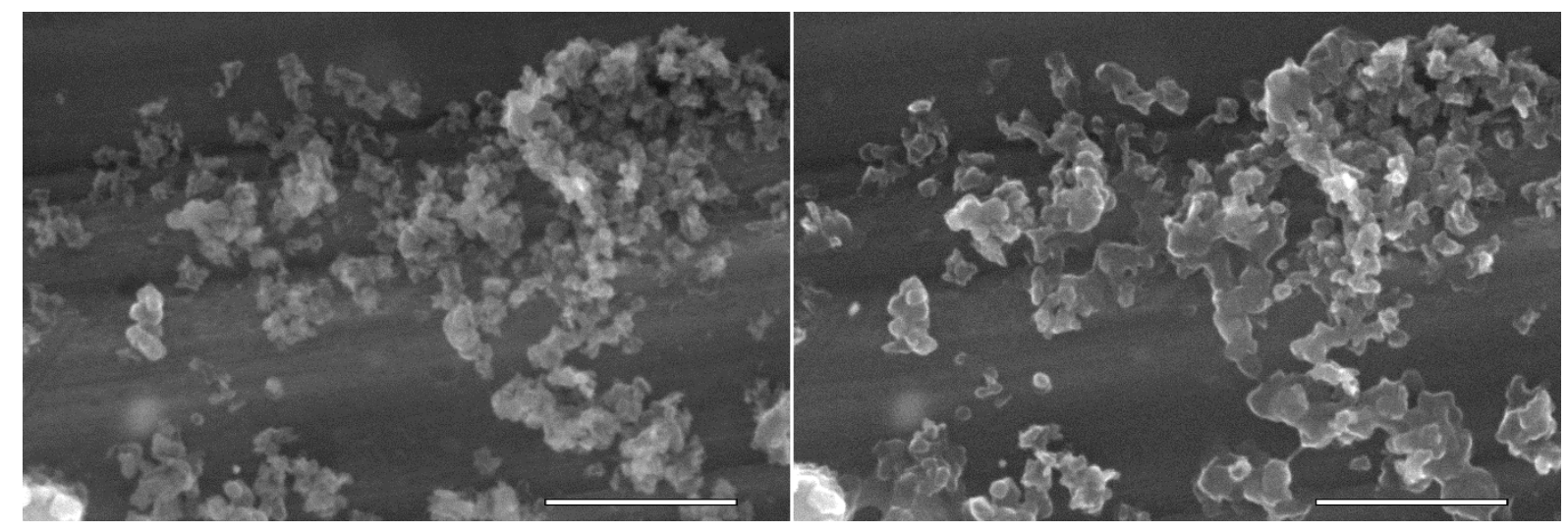

Figure 4: Environmental SEM images of MIL-53(MeOH)-Acti at 60\% relative humidity (left) and $80 \%$ relative humidity (right). Particles start to fuse at higher humidity levels. Scale bar: $2 \mu \mathrm{m}$
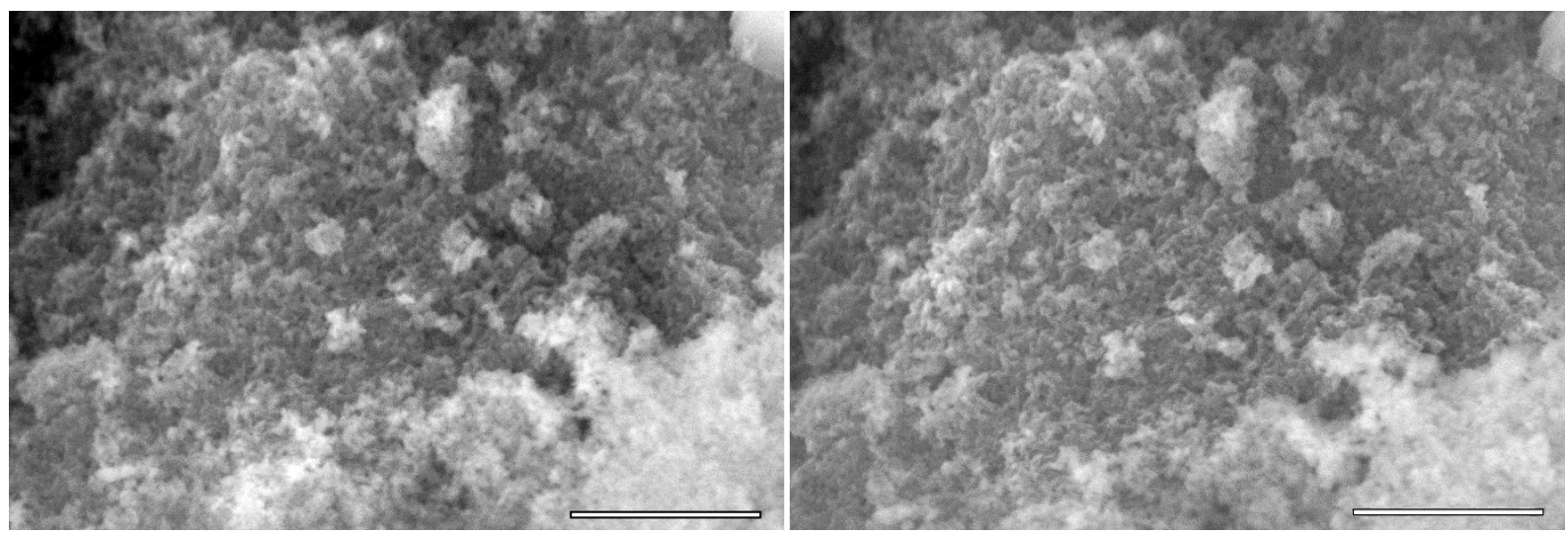

Figure 5: Environmental SEM images of MIL-53(DMF)-Acti at 60\% relative humidity (left) and $80 \%$ relative humidity (right). No change of the particles was observed. Scale bar: $2 \mu \mathrm{m}$

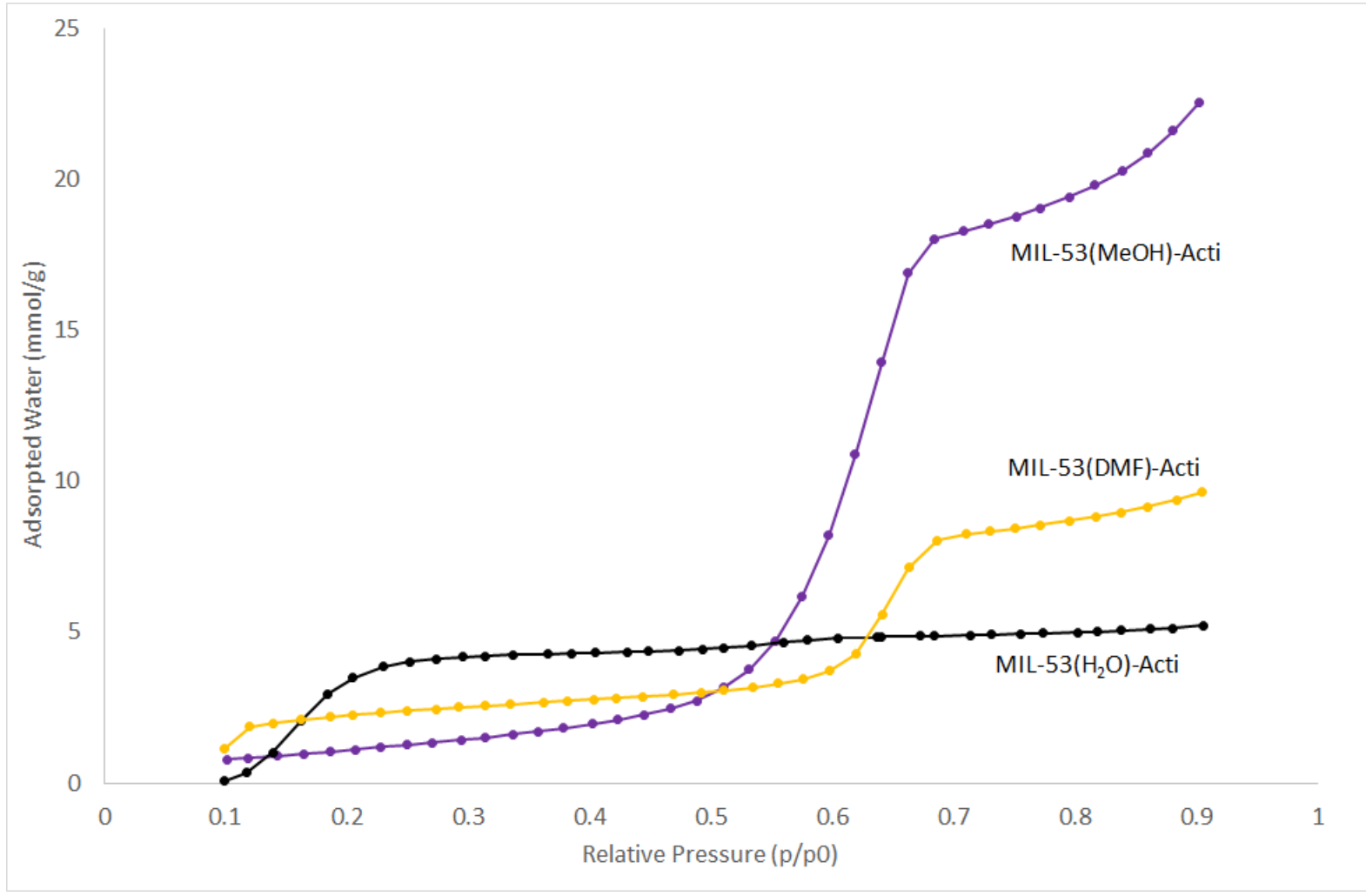

Figure 6: Water isotherm of MIL-53( $\left.\mathrm{H}_{2} \mathrm{O}\right)$-Acti (black), MIL-53(DMF)-Acti (yellow) and MIL-53(MeOH)-Acti (purple) measured at $25^{\circ} \mathrm{C}$ and ambient pressure. The isotherms were measured between 0.1 and $0.9 \mathrm{p} / \mathrm{pO}$. 